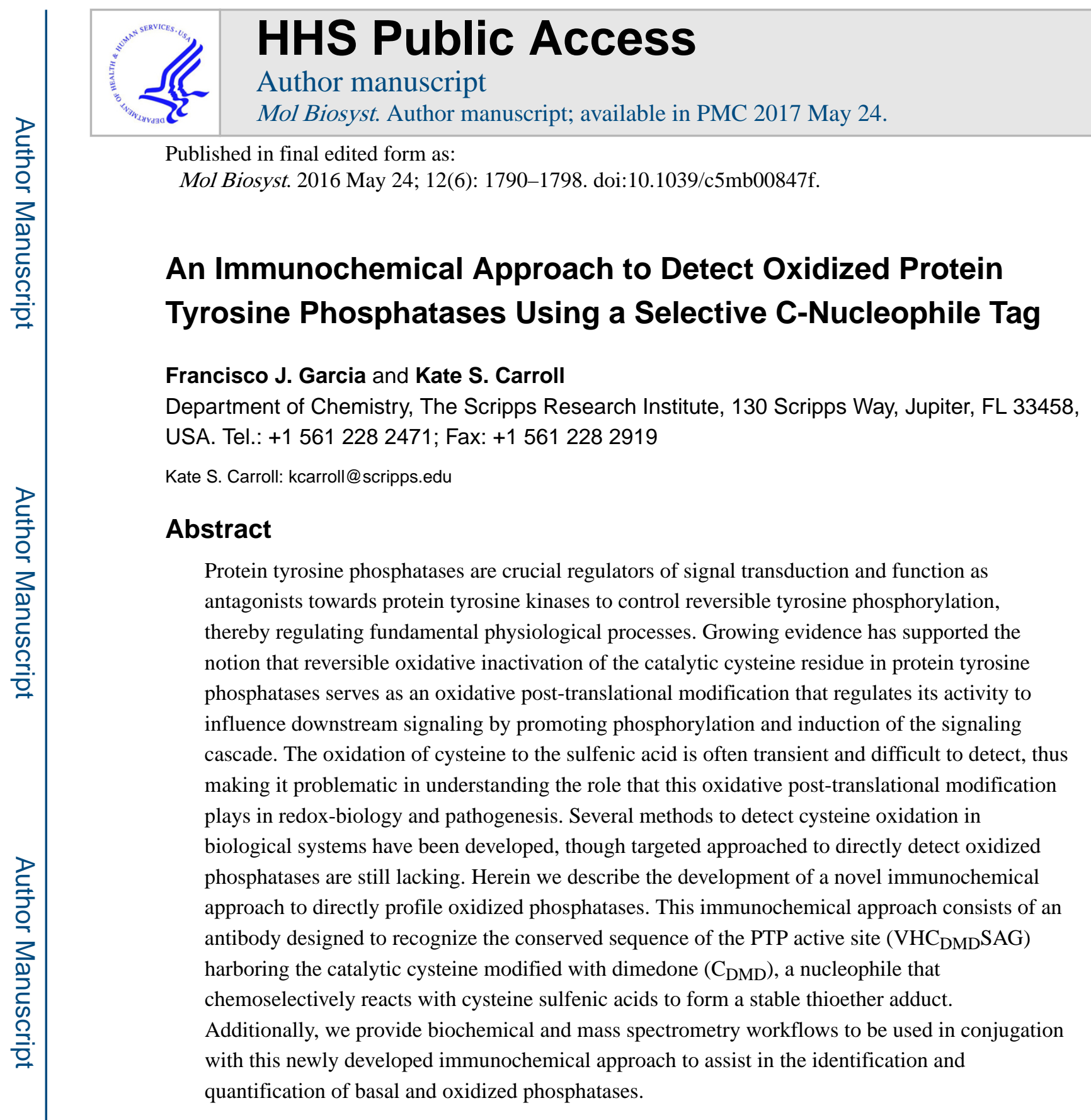

\title{
Table of Contents (TOC)
}

We report a simplified immunochemical approach to directly detect and quantify oxidized protein tyrosine phosphatases modified with dimedone.

\footnotetext{
Correspondence to: Kate S. Carroll, kcarrollescripps . edu.

$\dagger$ Electronic Supplementary Information (ESI) available: See DOI: 10.1039/x0xx00000x
} 


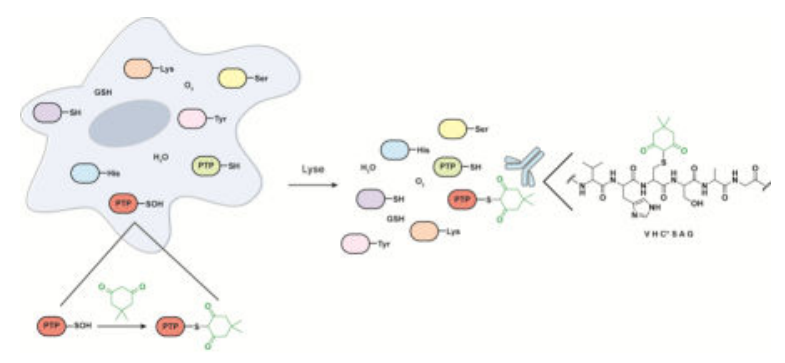

\section{Introduction}

Phosphorylation of tyrosine residues by protein tyrosine kinases (PTKs) is a key posttranslational modification that orchestrates many aspects of cellular signaling ranging from proliferation, differentiation, cell survival, metabolism, cell migration, and cell-cycle control. ${ }^{1}$ Tyrosine phosphorylation is tightly regulated by the opposing actions of protein tyrosine phosphatases (PTPs), which catalyze the hydrolytic dephosphorylation of phosphorylated tyrosine residues, thereby countering the activities of PTKs and maintaining a balance in cellular phosphorylation and signaling.

PTPs catalyze the dephosphorylation of phosphorylated tyrosine residues via a conserved catalytic cysteine residue, which exhibits a reduced $\mathrm{pK}_{\mathrm{a}}$ and exists as a stable thiolate as a consequence of being surrounded by basic amino acids that stabilize the anion. The unique architecture of the catalytic site renders the active site cysteine a potent nucleophile and facilitates PTPs to carry out their enzymatic function. PTPs have been shown to be tightly regulated by several mechanisms such as spatiotemporal expression, subcellular localization, proteolysis, dimerization, and post-translational modifications. ${ }^{2}$ Recent studies have shown that the reversible oxidation of PTPs has emerged as an important general post-translational regulatory mechanism for members of this enzyme family under physiologic and pathologic conditions. Oxidation of PTPs converts the catalytic cysteine to a sulfenic acid ( $\mathrm{SOH}$ ) and, depending on the architecture of the catalytic microenvironment, can rapidly rearrange to form a cyclic sulfenamide with the nitrogen of a neighboring amide peptide backbone residue or a disulfide bond with a nearby cysteine residue. ${ }^{3-7}$ The formation of either the intramolecular disulfides or cyclic sulfenamide within the phosphatase protects against overoxidation of the catalytic cysteine residue to the sulfinic $\left(\mathrm{SO}_{2} \mathrm{H}\right)$ or sulfonic acid $\left(\mathrm{SO}_{3} \mathrm{H}\right)$ oxoforms which are biologically irreversible.

A wide range of compounds have been shown to induce reversible oxidation of PTPs such as superoxide, hydroperoxides, peroxymonophosphate, hypothiocyanous acid,

pyrroloquinoline, peroxymonocarbaonate, peroxytetradecanoic acid, nitric oxide, hydroxyl radicals, and peroxidized lipids. ${ }^{8-17}$ Additionally, PTP oxidation has been shown to be an intrinsic component of cell signaling whereby production of reactive oxygen species (ROS) is triggered by the activation of many classes of cell surface receptors including receptor tyrosine kinases (RTKs), integrins, cytokine receptors, G-protein-coupled receptors, and Tand B-cell receptors. ${ }^{18-23}$ The most studied and biologically relevant oxidant is hydrogen peroxide $\left(\mathrm{H}_{2} \mathrm{O}_{2}\right)$ due to its stability and selective reactivity towards cysteine thiolates. ${ }^{24}$ The most relevant sources of ROS production are NADPH oxidase (Nox) enzymes following 
activation of various RTKs and the premature leakage of electrons from the respiratory chain of the mitochondria. Several studies have reported the oxidation of specific PTPs in response to different types of cell stimuli, including PTP1B in epidermal growth factor receptor signaling, SHP2 in platelet-derived growth factor signaling, both PTP1B and TCPTP in insulin signaling, and SHP1 in B-cell receptor signaling. ${ }^{18,19,25,26}$ Thus, these studies show that ROS acts as a second messenger leading to the transient reversible oxidation and inactivation of PTPs to sustain enhanced phosphorylation within signaling cascades.

Oxidative inactivation of protein tyrosine phosphatases constitutes a major regulatory mechanism of enzyme activity under physiologic and pathologic conditions. A lack of methods to monitor PTP oxidation has made it difficult to study their redox regulation within cells. ${ }^{27}$ Current approaches to monitor PTP oxidation are mainly indirect and are dependent on electrophilic species that covalently modify the cysteine thiols (Figure 1A). Iodoacetate (IAA) bearing radiolabeled, biotinylated, or fluorescent tags have been used to monitor PTP oxidation whereby a decrease in the incorporation of the tagged-IAA is used to assess whether the phosphatase had been oxidized upon growth factor stimulation. ${ }^{19,}$ 21, 28 Since you are monitoring a loss of labeling as the PTP becomes oxidized, the major disadvantage of using tagged-IAA are sensitivity issues. A modified cysteinyl-labeling assay was developed to remedy the limitations of the IAA-tagged approach and relied on a biotinylated thiol-alkylating (IAP-biotin) reagent to indirectly monitor PTP oxidation. The assay consists of three steps: (i) alkylation of active-site cysteine residues of PTPs that had not been oxidized; (ii) reduction and reactivation of reversibly oxidized PTPs; and (iii) alkylation of the aforementioned reduced PTPs with IAP-biotin. ${ }^{29}$ The modified cysteinyllabeling assay is not a targeted approach and suffers from high background of non-PTP proteins and low sensitivity.

Other indirect approaches, such as the in-gel phosphatase assay, have been developed to permit the identification of oxidized PTPs (Figure 1A). This assay relies on the alkylation of thiols that have not been oxidized within a cell lysate. The PTPs that have been oxidized are protected from alkylation and the lysate is subsequently resolved by SDS-PAGE containing a radioactively labeled substrate. The resolved oxidized PTPs are reactivated and refolded in the presence of reducing agents and their detection is based on reactivity with the radiolabeled substrate. ${ }^{18,30}$ The in-gel phosphatase assay has limitations and is biased towards non-membrane bound phosphatases and is not quantifiable.

Immunochemical strategies have also been developed to indirectly detect oxidation of PTPs. Antibodies, generated towards the conserved catalytic motif of PTPs ([I/

V]HCSXGXGRS[S/T]G) bearing a sulfonic acid epitope on the catalytic cysteine, can be used to detect oxidized PTPs. The detection strategy requires: (i) capping of free thiols with alkylating reagents, (ii) PTP immunoprecipitation, (iii) reduction of oxidized PTP, and (iv) treatment of PTP with pervanadate (PV) to generate the hyperoxidized $\mathrm{SO}_{3} \mathrm{H}$ epitope (Figure 1A). Applications of this oxPTP antibody was used to show that UV-irradiation of cells could induce preferential oxidation of the second catalytic domain of RPTPa and monitor alterations in oxidation of SHP2 after treatment with platelet derived growth factor (PDGF). ${ }^{31,} 32$ Additionally, global quantification proteomic approaches have been combined with this immunochemical approach to assess PTP oxidation. ${ }^{33}$ The main limitations of this 
immunochemical approach is that it does not differentiate between phosphatases regulated by reversible oxidation from those that are inherently overoxidized. Moreover, the workflow for this immunochemical assay is cumbersome and may lead to false-positive results.

The detection of oxidized PTP via indirect methods require a workflow in which cells are lysed followed by subsequent manipulation of the samples. Cell lyses prior to capture of oxidized PTPs may lead to artificial oxidation due to the disruption of the various redox compartments within the cell. Direct approaches to monitor oxidized PTPs have been developed and circumvent issues with artificial oxidation brought about by cell lyses and egregious protein manipulation. Nucleophiles such as 5,5-dimethyl-1,3-cyclohexanedione, also known as dimedone (DMD), have been shown to selectively react with protein sulfenic acids to generate a stable thioether adduct (Figure 1B). ${ }^{34}$ Bifunctional chemical probes that incorporate reporter/affinity tags on the DMD nucleophile allows for the direct detection of oxidized proteins using a single reagent and has had success in the detection and quantification of various oxidized proteins, including PTPs. ${ }^{35}$ Several probes to exclusively monitor PTP oxidation have been developed. These PTP redox-based probes (RBPs) are composed of: (i) a DMD-based nucleophile which chemoselectively reacts with the oxidized catalytic cysteine; (ii) a module that directs binding to the PTP catalytic site; and (iii) a reporter tag used for the identification, purification, or direct visualization of the labeled protein. ${ }^{36}$ These RBPs have been shown to detect basal and growth factor induced oxidized PTP1B in cells stimulated with insulin and have potential to be used to study redox regulation of phosphatases via global proteomic assessment. ${ }^{37}$

DMD-specific antibodies, generated from a thiodimedone hapten consisting of the DMD thioether moiety on a cystamine backbone, are available to directly monitor protein oxidation via the protein-S-DMD adduct. ${ }^{38}$ These antibodies have been applied to monitor PTP oxidation in vitro but may also be used to detect oxidized phosphatases in DMD-treated cells via immunoprecipitation of the desired PTP followed by immunoblotting. ${ }^{37}$ Additionally, conformation sensing antibodies have been developed to directly detect unique conformational changes associated with oxidation of PTP1B to the cyclic sulfenamide. ${ }^{39}$ Though the conformation sensing antibody provides a direct approach to monitor PTP oxidation, they are specific for a single phosphatase (PTP1B) and may not be used to monitor oxidation of the entire PTP family.

Oxidation of PTPs has emerged as an important mechanism for regulation of PTP activity and several approaches for oxPTP detection exist, improvements of the tools available are necessary and may aid in the investigation of PTP redox regulation. Herein we provide the development of an immunochemical approach that directly detects oxidized PTPs (Figure 1B). We also provide biochemical and mass spectrometry workflows to assist in the identification and quantification of basal and oxidized PTPs.

\section{Materials and Methods}

\section{Synthesis of $\mathrm{VHC}_{\mathrm{DMD}} \mathrm{SAG}$ hapten and generation of antibodies}

The heptapeptide was synthesized on solid phase using standard Fmoc main chain and Boc/Trt side chain protection chemistry. The VHCSAG peptide was treated with bromo- 
dimedone to generate the $\mathrm{VHC}_{\mathrm{DMD}} \mathrm{SAG}$ modified peptide. $\mathrm{KLH}$ (Keyhole Limpet Hemocyanin) protein was activated by adding sulfo-SMCC (sulfosuccinimidyl 4-[Nmaleimidomethyl]cyclohexane-1-carboxylated), a hetero-bifunctional crosslinker containing an amine-reactive $\mathrm{N}$-hydroxysuccininmide and a sulfhydryl-reactive malemide group. The hapten was conjugated to succinylated KLH overnight at $\mathrm{pH} 8$ and the product was then purified over a PD10 gel filtration column (GE Healthcare). Two New Zealand White (specific pathogen-free grade) rabbits were immunized subcutaneiously with conjugate in a 50:50 emulsification with adjuvant [either Freunds complete (FCA) or Freunds incomplete (FIA) according to the following schedule: day 0 boost (FCA), day 14 boost (FIA), and day 28 boost (FIA). The rabbits were bled on days 35 and 40 after the primary boost, the red blood cells spun out by centrifugation, and the remaining antisera was IgG purified using a Nab Protein A Plus Spin Kit (Thermo Scientific).

\section{Preparation of recombinant PTP1B and VHR}

The construct of PTP1B was a kind gift from Kent S. Gates and was expressed and purified as previously described. ${ }^{13}$ VHR was cloned into an expression vector containing a TEV cleavable $6 \mathrm{x}$-His tag via ligation independent cloning (LIC) techniques. The cDNA for VHR (HsCD00000599) and the LIC vector, pMCSG28 (EvNO00340964) were purchased from DNAsu. The VHR DNA fragment was amplified with primers: 5 GTCTCTCCCATGATGTCGGGCTCGTTCGAC - 3; and 5 GGTTCTCCCCAGCCTAGGGTTTCAACTTCCCCTC - 3; using standard PCR methods. The PCR product was purified using the Wizard SV gel and PCR cleanup system (Promega). The PCR products were treated with T4 DNA polymerase (New England BioLabs) and dTTP. The pMCSG28 vector was linearized by digestion with SmaI, purified using the Wizard SV gel and PCR clean-up system, and then treated with T4 DNA polymerase and dATP. The PCR product was annealed to vector DNA by mixing $3 \mu \mathrm{L}$ T4-treated pMCSG28 with $2 \mu \mathrm{L}$ T4-treated PCR product and incubated on ice for 30 minutes, followed by transformation into E. coli. After sequencing verified successful cloning, the vector was transformed into BL21(DE3) cells, grown in LB media, induced with IPTG, purified via NiNTA and size exclusion chromatography. Recombinant CDC25A, SHP2, PTEN, MKP1, and IF2 were purchased from Enzo Life Sciences.

\section{Chemical modification of recombinant phosphatases}

Stocks of the phosphatases were treated with $50 \mathrm{mM}$ DTT for 20 minutes on ice, followed by removal of DTT via buffer exchange with Nap-5 columns (GE Healthcare) preequilibrated with labeling buffer (50 mM HEPES, $100 \mathrm{mM} \mathrm{NaCl}, 1 \mathrm{mM}$ EDTA, pH 7.0). Protein concentrations were determined by A280 using a NanoDrop (Thermo). $10 \mu \mathrm{M}$ PTP was treated with $10(100 \mu \mathrm{M})$ or more equivalences of $\mathrm{H}_{2} \mathrm{O}_{2}$ for $1-2$ hours in the presence of varying concentrations of nucleophile. Excess reagent was removed by passing the sample through a P-30 column (BioRad) pre-equilibrated with $25 \mathrm{mM} \mathrm{ABC}$. The commercially purchased recombinant phosphatases were buffer exchanged into aforementioned labeling buffer using a pre-equilibrated P-6 column (Bio-Rad). $0.1 \mu \mathrm{g} / \mu \mathrm{L}$ of each recombinant phosphatase was treated with $100 \mu \mathrm{M} \mathrm{H}_{2} \mathrm{O}_{2}$ in the presence and absence of $10 \mathrm{mM}$ DMD for 1 hour. The samples were then prepped for Western blot analysis. 


\section{Ratiometric analysis of basal and ROS-mediated oxidized VHR}

Recombinant VHR was prepared and buffer exchanged as mentioned above. VHR was either treated with $10 \mathrm{mM}$ light DMD in the absence of $\mathrm{H}_{2} \mathrm{O}_{2}$ or with $10 \mathrm{mM}$ heavy DMD in the presence of varying concentrations of $\mathrm{H}_{2} \mathrm{O}_{2}$ for 2 hours at room temperature. Afterwards, the samples were passed through a pre-equilibrated P-30 column to remove excess reagent and then mixed at a 1:1 ratio. The samples were digested in solution using trypsin overnight, desalted using C-18 columns (The Nest Group), and immunoprecipitated with the PTP-SDMD antibody. The samples were then subjected to MS/MS analysis.

\section{Isotope-coded dimedone and iododimedone (ICDID) for quantification of protein sulfenic acid}

Recombinant VHR was prepared and buffer exchanged as mentioned above. VHR was treated with $10 \mathrm{mM}$ heavy dimedone in the presence of varying concentrations of $\mathrm{H}_{2} \mathrm{O}_{2}$ for 2 hours at room temperature. Afterwards, the protein mixture was treated with $10 \mathrm{mM}$ iododimedone for 1 hour at room temperature. The protein mixture was then subjected to an in-solution digestion using trypsin overnight. The following day, the peptides were desalted using a C-18 column (The Nest Group) and immunoprecipitated with the PTP-S-DMD antibody. The samples were then subjected to MS/MS analysis.

\section{Immunoprecipitation of PTP-S-DMD peptides}

The tryptic digests were desalted using C-18 columns (The Nest Group) as described above. The desalted digest was concentrated to dryness and resuspended in $1 \mathrm{~mL}$ of $1 \times$ PBS. PTPS-DMD antibody $(5 \mu \mathrm{L})$ was added to the peptide suspension and allowed to mix overnight at $4{ }^{\circ} \mathrm{C}$ on an end-over-end rocker. The following day, the peptide mixture was treated with $20 \mu \mathrm{L}$ protein A/G PLUS-Agarose (Santa Cruz Biotechnology) and allowed to mix at $4{ }^{\circ} \mathrm{C}$ for 3 hours on an end-over-end rocker. The immunocomplex was collected by centrifugation at $1000 \times \mathrm{g}$ for 2 minutes, washed with 1x PBS ( 3 x's), washed with $\mathrm{H}_{2} \mathrm{O}$ ( 3 x's), and eluted by incubation with $0.15 \%$ formic acid $(80 \mu \mathrm{L})$ for 10 minutes ( $3 \mathrm{x}$ 's). The eluted peptides were concentrated to dryness, respusended in $50 \mu \mathrm{L} 0.1 \%$ formic acid and analyzed by MS.

\section{Mass spectrometry analysis}

Samples were analyzed by liquid chromatography-tandem MS (LC-MS/MS) using an EASY-nLC II system coupled to a linear ion trap mass spectrometer model LTQ (Thermo Fisher Scientific). Peptides were concentrated and desalted on a RP precolumn $(0.1 \times 20$ mm EASY-column, Thermo Fisher Scientific) and on-line eluted on an analytical RP column $(0.075 \times 100 \mathrm{~mm}$ EASY-column, Thermo Fisher Scientific), operating at 300 $\mathrm{nL} / \mathrm{min}$ and using a gradient of $5 \%-90 \% \mathrm{~B}$ over 45 minutes [solvent A: $0.1 \%$ formic acid $(\mathrm{v} / \mathrm{v})$; solvent B: $0.1 \%$ formic acid $(\mathrm{v} / \mathrm{v})$ in $80 \%$ acetonitrile].

\section{Detection of oxSHP2 in A431 cells}

A431 (ATCG) cells were maintained at $37{ }^{\circ} \mathrm{C}$ in a $5 \% \mathrm{CO}_{2}$ humidified atmosphere. Cells were cultured in high glucose DMEM (Corning) containing 10\% FBS (Invitrogen), 1\% GlutaMax (Invitrogen), 1\% MEM nonessential amino acids (Invitrogen), and 1\% penicillinstreptomycin (Invitrogen). For EGF treatment, cells were serum starved for 16 hours prior to 
experimentation. Cells were grown to $80 \%$ confluency prior to serum starvation. A431 cells were stimulated with $100 \mathrm{ng} / \mathrm{mL}$ EGF (or varying concentrations of EGF) for 5 minutes. Afterwards, cells were washed with PBS ( 3 x's) and treated with $10 \mathrm{mM}$ DMD (or varying concentrations of DMD) for 1 hour at $37{ }^{\circ} \mathrm{C}, 5 \% \mathrm{CO}_{2}$. Cells were washed with PBS ( 3 x's) to remove excess nucleophile and the cells were harvested in a NP-40 lysis buffer [50 mM Tris-HCl pH 8.0, $137 \mathrm{mM} \mathrm{NaCl}, 10 \%$ glycerol, $1 \%$ NP-40, $50 \mathrm{mM} \mathrm{NaF,} 10 \mathrm{mM} \beta$ glycerolphosphate, $1 \mathrm{mM}$ sodium vanadate, $1 \mathrm{x}$ EDTA-free protease cocktail inhibitors (Roche), and $200 \mathrm{U} / \mathrm{mL}$ catalase (Sigma)]. After 20 minutes incubation on ice with frequent mixing, cell debris was removed by centrifugation at $14000 \mathrm{rpm}$ at $4{ }^{\circ} \mathrm{C}$ for 15 minutes. Protein concentrations were determined by BCA assay (Pierce). SHP2 was immunoprecipiated from $1 \mathrm{mg}$ of cell lysate.

\section{Western blot analysis}

Protein samples were resolved by SDS-PAGE using Mini-Protean TGX 4-15\% Tris-Glycine gels (BioRad) and transferred to a polyvinylidene difluoride (PVDF) membrane (BioRad). After transfer, the PVDF membrane was blocked with 3\% BSA in TBST for 1 hour at room temperature. The membrane was washed with TBST (3X) and immunoblotting was performed with the following primary and secondary antibodies at the indicated dilutions: PTP-S-DMD AB (1:200 - 1:1000), SHP2 (Santa Cruz Biotechnology; 1:200), goat antirabbit IgG-HRP (Calbiochem, 1:1000 - 1:50000), and rabbit anti-mouse IgG-HRP (Invitrogen, 1:35000) The PVDF membranes were washed with TBST (3X) and developed with ECL Plus chemiluminescence (Pierce) and imaged by film. Equal loading of recombinant phosphatases were assessed by treating the membrane with a solution of 1:1 $\mathrm{MeOH}: \mathrm{R}-250$ Coomassie blue for ten minutes, then allowing membrane to dry.

\section{Results and Discussion}

To detect $\mathrm{H}_{2} \mathrm{O}_{2}$ mediated oxidized PTPs, we envisioned an enhancement of both the oxPTP and the thiodimedone-specific antibody approach via complementation of the conserved active site peptide, representative of the classical PTPs, with the DMD moiety (Figure 1B). ${ }^{31,38}$ This enhanced antibody strategy will allow for improved detection of the oxidation-induced inactivation of PTPs. We designed and synthesized the PTP active site peptide (VHCSAG) via standard peptide coupling strategies. The peptide was then reacted with Bromo-DMD to give the desired conserved catalytic peptide harboring the stable DMD thioether adduct. The resulting peptide was modified to a five-carbon linker to incorporate the keyhole limpet hemocyanin (KLH). The resulting hapten-protein conjugate was used to elicit rabbit a-hapten-Ig gamma ( $\operatorname{IgG})$.

The human genome contains a set of 107 genes that encode for PTPs. This large enzyme family is subdivided into four classes based on their substrate specificity and amino acid sequence at the catalytic domain. Class I PTPs are comprised of classical and dualspecificity phosphatases, which dephosphorylate phospho-tyrosine and phospho- serine/ threonine/tyrosine residues respectively. The class II phosphatases consist of low molecular weight PTPs (LMPTPs) and are encoded by the ACP1 gene. The class III phosphatases are made up of three genes encoding the Cdc25 family members. Finally, class IV are aspartic 
acid-based PTPs and are encoded by the four eyes absent homologue (EYA) gene. We expected that the antibody would be useful for the global detection of DMD-modified oxPTPs. To test this, we took various recombinant PTPs that were representative of the three cysteine-based PTP classes (I, II, and III) within the PTP superfamily and subjected them to oxidation in the presence or absence of DMD. Western blot analysis demonstrated a clear signal for all the PTPs that had been treated with $\mathrm{H}_{2} \mathrm{O}_{2}$ in the presence of DMD (Figure 2). These results suggest that the antibody may be suitable for global detection of DMDmodified oxPTPs. Future studies were performed on PTP1B, VHR, and SHP2 which all showed robust detection with the antibody.

Initial studies with recombinant PTP1B were performed to determine whether the antibody could detect DMD-modified oxPTPs in a dose- and time-dependent manner. $10 \mu \mathrm{M}$ PTP1B was treated with varying concentrations of DMD in the presence of 10 equivalences (100 $\mu \mathrm{M}) \mathrm{H}_{2} \mathrm{O}_{2}$. The protein was resolved by SDS-PAGE and subjected to Western blot analysis. The immunoblot demonstrated that the antibody is able to detect DMD-modified oxPTP1B in a dose- (Figure 3A) and time-dependent fashion (Figure 3C). Densitometric analysis of the dose- (Figure 3B) and time-dependent (Figure 3D) Western blots reveal robust detection of this oxidative post-translational modification. We next evaluated the specificity of the antibody by determining whether it can fully discriminate between oxPTPs that have been modified with DMD from non-PTP redox-sensitive proteins, such as the antioxidizing enzyme - glutathione peroxidase 3 (Gpx3), that have also been shown to react with DMD. We treated Gpx3 with varying concentrations of DMD in the presence of $\mathrm{H}_{2} \mathrm{O}_{2}$ followed by SDS-PAGE and Western blot analysis and revealed that the antibody is solely selective for oxPTPs modified with DMD (Supplementary Figure 1A). Densitometric analysis confirmed only background (Supplementary Figure 1B). Finally, we tested the antibodies ability to differentiate between detection of DMD-modified oxPTPs from other nucleophiles that are structurally analogous to DMD. We treated recombinant PTP1B with $\mathrm{H}_{2} \mathrm{O}_{2}$ and varying concentrations of indandione (IND) and piperdinedione (PRD), both of which showed enhanced reactivity towards a small molecule $\mathrm{SOH}$ model as compared to DMD, and monitored their detection with the antibody. ${ }^{40,41}$ We observed detection of DMD-modified oxPTP1B only and no detection with the DMD nucleophilic isosteres (Supplementary Figure 1C). Taken together, these findings suggest that the antibody is a practical tool with high affinity and selectivity for detecting DMD-modified oxPTPs.

We next proposed to justify the usefulness of the antibody for facile detection of oxPTPs in cells. Previous antibody-based approaches that were developed to detect reversibly oxPTP used a monoclonal antibody that recognized the conserved sequence of PTPs bearing the catalytic cysteine terminally oxidized to the $\mathrm{SO}_{3} \mathrm{H}$. This approach required a cumbersome workflow in which cell lysates were treated with alkylating reagents to cap free thiols, followed by treatment with dithiothreitol (DTT) to regenerate the reversibly oxidized PTPs, and finally treatment with pervanidate to generate the $\mathrm{SO}_{3} \mathrm{H}$. We devised a scheme in which the antibody would allow for a simplistic workflow in the detection of oxPTPs

(Supplementary Figure 2A). We envisioned that intact cells would be stimulated with growth factors to prompt the production of endogenous $\mathrm{H}_{2} \mathrm{O}_{2}$ associated with various cell signaling pathways. Following growth factor stimulation, the cells would be treated with DMD to trap the sulfenylated PTPs. Next, the cells would be lysed and the desired PTP would be 
immunoprecipiated and analyzed by Western blot analysis for DMD-modified oxPTPs using the PTP-S-DMD specific antibody. To evaluate this approach, we transfected CHO/hIRc cells with HA-tagged PTP1B (pJ3H-PTP1B) and subsequently stimulated the transfected cells with $100 \mathrm{nM}$ insulin. Following insulin stimulation, the cells were treated with or without $10 \mathrm{mM}$ DMD, lysed, and enriched for HA-PTP1B. Western blot analysis demonstrates the ease at which this antibody allows for the detection of oxPTP1B (Figure 3E). Densitometric quantification illustrated a two-fold increase of oxPTP1B after insulin stimulation (Figure 3F). Taken together, these results indicate that the PTP-S-DMD antibody offers a more rapid and simplistic approach for the direct detection of oxPTPs from a complex cellular milieu.

SHP2 directly interacts with the epidermal growth factor receptor (EGFR) and previous studies have shown that SHP2 is highly susceptible to reversible oxidative inactivation upon epidermal growth factor (EGF) stimulation. ${ }^{42}$ We therefore wanted to determine whether the antibody would be able to detect SHP2 oxidation upon stimulation with EGF in cells. A431 cells were stimulated with $100 \mathrm{ng} / \mathrm{mL}$ EFG followed by incubation with increasing concentrations of DMD. Western blot analysis of the immunoprecipitated SHP2 revealed a modest increase in DMD-modified SHP2 in the presence of $10 \mathrm{mM}$ DMD (Supplementary Figure 2B). Densitometric quantification of the blot showed a two-fold increase in DMDmodified oxSHP2 after stimulation with EGF in the presence of $10 \mathrm{mM}$ DMD (Supplementary Figure 2C). Time-dependent analyses were also performed with immunoprecipitated oxSHP2 and were in agreement with our in vitro studies (Supplementary Figure 2D). Stimulation of A431 cells with varying concentrations of EGF revealed a dose-dependent enhancement of DMD-modified oxSHP2 detection (Supplementary Figure 2E). Densitometric quantification of the blot showed a two- to threefold increase in the levels of oxSHP2 in respects to increasing concentration of EGF (data not shown). In 1998, Lee et al. found that PTP1B is transiently inactivated after stiumulation of cells with EGF and determined that oxidative inactivation modulates the steady-state levels of phosphorylation necessary for growth stimulation. ${ }^{19}$ To show the broad utility of our antibody, we performed a similar experiment in COS1 cells transfected with HA-PTP1B. The stimulation of COS1 cells with various concentrations of EGF displayed an expected dose-dependent increase in DMD-modified oxPTP1B (Supplemental Figure 2F). These results provide evidence for the usefulness of this immunochemical approach for the facile direct detection of oxidized phosphatases within a complex cellular setting.

Apart from immunochemical detection of oxidized phosphatases, we anticipated that the antibody would be useful for enriching DMD-modified PTP catalytic peptides for subsequent proteomic analysis. We initially tested whether the antibody could selectively pull down the DMD-modified catalytic peptide hapten. A solution of the hapten was prepared in PBS and treated with or without the antibody and allowed to mix overnight at $4{ }^{\circ} \mathrm{C}$. The following day, the immunocomplex was pulled down with protein $\mathrm{A} / \mathrm{G}$ agarose, washed extensively before eluting with $0.15 \%$ trifluoroacetic acid (TFA), and analyzed by LC/MS/MS. We observed a peak with a mass of $711.31 \mathrm{Da}$, corresponding to the $[\mathrm{M}+\mathrm{H}]^{+1}$ $\mathrm{m} / \mathrm{z}$ of $\mathrm{VHC} * \mathrm{SAG}\left(\mathrm{C}^{*}=\right.$ DMD modified). MS/MS confirmed the sequence of the peptide (Supplemental Figure 3A). In the absence of the antibody, no peaks were observed indicating that the hapten does not bind non-specifically to the IgG agarose beads. Thus the 
preliminary results suggest that the antibody is suitable for immunoprecipitating DMDmodified peptides.

We next sought to determine whether the antibody could enrich the DMD-modified catalytic peptide from a complex mixture. We choose VHR as a model system since previous studies indicated that oxidized VHR forms a stable $\mathrm{SOH}^{43}$ We therefore treated recombinant VHR with varying concentrations of $\mathrm{H}_{2} \mathrm{O}_{2}$ in the presence of $10 \mathrm{mM}$ DMD, followed by insolution digestion with trypsin, and enrichment of DMD-modified peptides with the antibody. We observed a $\mathrm{H}_{2} \mathrm{O}_{2}$ dose-dependent increase in the intensity of DMD-labeled VHR catalytic peptide (Supplemental Figure 3B). MS/MS analysis of the peak at $432.73 \mathrm{Da}$, which corresponds to the $[\mathrm{M}+\mathrm{H}]^{+2} \mathrm{~m} / \mathrm{z}$, confirmed the sequence of the catalytic peptide (Supplemental Figure 3B).

Isotopically labeled $\mathrm{SOH}$ probes have previously been developed to assist in quantifying the extent of protein $\mathrm{SOH}$ modification during growth factor mediated $\mathrm{H}_{2} \mathrm{O}_{2}$ production under normal and disease states. ${ }^{44,}{ }^{45}$ We envisioned that the antibody may be useful for differentiating proteins that are oxidized under basal conditions from those that are oxidized during growth factor induced $\mathrm{H}_{2} \mathrm{O}_{2}$ production (Figure 4A). We treated separate samples of VHR with either $10 \mathrm{mM}$ of light-DMD or heavy-DMD in the absence or presence of $\mathrm{H}_{2} \mathrm{O}_{2}$, respectively. We then pooled the two samples together, generated a tryptic digest, immunoprecipitated the DMD-modified catalytic peptides, and subsequently analyzed the immunoprecipitated peptides by LC/MS/MS. We observed the light-DMD peak at $\mathrm{m} / \mathrm{z}$ $432.74[\mathrm{M}+\mathrm{H}]^{+2}$ and heavy-DMD at $\mathrm{m} / \mathrm{z} 435.76[\mathrm{M}+\mathrm{H}]^{+2}$. MS/MS of heavy-DMD modified VHR confirmed the sequence and area under the curve (AUC) calculations indicated $\sim 5.3 \%$ basal oxidized VHR (Figure 4B).

Furthermore, we proposed that the antibody would be useful for distinguishing between SOH modified PTPs and PTPs that reside in the thiol state. We therefore utilized the isotope coded dimedone and iododimedone (ICDID) method for the quantitative analysis of protein SOH modification in conjugation with the PTP-S-DMD approach to specifically monitor the extent of oxPTP formation in order to determine the extent or fraction of $\mathrm{SOH}$ modified PTPs (Figure 5A). ${ }^{46}$ Here we treated VHR with varying concentrations of $\mathrm{H}_{2} \mathrm{O}_{2}$ in the presence of $10 \mathrm{mM}$ heavy DMD to intercept any oxidized VHR. Afterwards, we treated the same sample with $10 \mathrm{mM}$ iododimedone to trap any cysteine thiol that had not been oxidized. The samples were digested, DMD-modified catalytic peptides were immunoprecipitated with the antibody via immunoaffinity enrichment, and subsequently analyzed by LC/MS/MS. We observed a change in the ratio of heavy DMD modified VHR from light DMD modified VHR with increasing concentrations of $\mathrm{H}_{2} \mathrm{O}_{2}$ (Figure 5B). This suggested that with increasing concentrations of $\mathrm{H}_{2} \mathrm{O}_{2}$ more of the PTP exists as a $\mathrm{SOH}$ and not as a free thiol. Taken together, these studies indicate that the antibody may be a practical tool for identifying and quantifying oxidized phosphatases.

\section{Conclusion}

While approaches to identify specific oxidized PTPs are available, it has been difficult to develop general methods that detect and quantify any reversible oxidized PTPs in different 
physiological and pathological states. Therefore, tools that can directly detect $\mathrm{SOH}$ modified phosphatases through a simplistic workflow are necessary to help further our understanding of how this oxidative post-translational modification plays a role in disease states. The majority of current methods indirectly monitor phosphatase oxidation. Small molecules that chemoselectivley react with protein SOH have been developed to directly monitor PTP oxidation. These compounds are chemically selective for $\mathrm{SOH}$, bear binding modules to direct them to the phosphatase super family, and are cell permeable. ${ }^{36,37}$ To this end, we have expanded the toolkit for the direct detection of this oxidative post-translational modification via the development, design, and implementation of an immunochemical approach. This immunochemical strategy was inspired from several known methods that detect oxidized phosphatases and improved on for facile implementations in workflows for the selective detection of oxidized phosphatases. Initial studies performed in vitro demonstrated that the antibody could detect DMD-modified phosphatases in a time- and dose-dependent fashion. Additionally, the antibody was shown to be selective for phosphatases modified with DMD as opposed to other proteins that are susceptible to oxidation and reaction with DMD. Furthermore, the antibody was shown to solely recognize the DMD epitope as opposed to other nucleophiles (IND or PRD) that share structural similarities but exhibit enhanced reactivity for $\mathrm{SOH}$. Since all phosphatases contain a conserved catalytic domain, we confirmed that this immunochemical approach would be useful for global detection of oxidized PTPs. We then used the antibody to detect endogenous levels of oxidized phosphatases in cells. A431 cells, stimulated with varying concentrations of EGF, displayed a dose-dependent increase in the levels of oxidized SHP2. Finally, we proposed that this immunochemical approach would be useful for enrichment and subsequent proteomic analysis. A major strength of this approach is that it can be coupled to other previously developed technologies for quantification of protein sulfenic acids. Using heavy DMD, we were able to differentiate between basal oxidized PTPs from those that were oxidized in the presence of $\mathrm{H}_{2} \mathrm{O}_{2}$. Moreover, implementation of the ICDID method allowed for the quantification of protein $\mathrm{SOH}$ in the presence of various concentrations of $\mathrm{H}_{2} \mathrm{O}_{2}$. This new method for the direct detection of $\mathrm{SOH}$ modified phosphatases provides a powerful means to detect changes in cysteine oxidation in this protein family in vivo and should find a wide variety of applications for the study of biological processes revolving around redox signaling pathways that are central to human health and disease. Future applications of this approach can be used for detection, identification, and quantification of redox regulated phosphatases in cells during various cell signaling events and may be useful for a systems biology understanding of redox signaling. Recently its been shown that PTKs are susceptible to oxidative modification and our immunochemical approach can be extended for the development of PTK-S-DMD antibodies to assist in detecting redox-regulated kinases. ${ }^{42}$ Additionally, with the discovery of highly reactive chemoselective nucleophiles towards $\mathrm{SOH}$, we may be able to develop $2^{\text {nd }}$ generation immunochemical approaches which harbor these more reactive nucleophiles on conserved peptide sequences for key signaling proteins such as PTPs and PTKs. ${ }^{40,} 41$

\section{Supplementary Material}

Refer to Web version on PubMed Central for supplementary material. 


\section{Acknowledgments}

This work was supported by the National Institute of Health (R01-GM102187 and R01-CA174864 to K.S.C.). We would like to thank Pablo Martinez-Acedo (Scripps) for helpful discussions and mass spectrometry support and Kent S. Gates (U. Missouri) for the PTP1B constructs.

\section{References}

1. Lemmon MA, Schlessinger J. Cell. 2010; 141:1117-1134. [PubMed: 20602996]

2. den Hertog J, Ostman A, Bohmer FD. The FEBS journal. 2008; 275:831-847. [PubMed: 18298791]

3. Salmeen A, Andersen JN, Myers MP, Meng TC, Hinks JA, Tonks NK, Barford D. Nature. 2003; 423:769-773. [PubMed: 12802338]

4. van Montfort RL, Congreve M, Tisi D, Carr R, Jhoti H. Nature. 2003; 423:773-777. [PubMed: 12802339]

5. Yang J, Groen A, Lemeer S, Jans A, Slijper M, Roe SM, den Hertog J, Barford D. Biochemistry. 2007; 46:709-719. [PubMed: 17223692]

6. Chen CY, Willard D, Rudolph J. Biochemistry. 2009; 48:1399-1409. [PubMed: 19166311]

7. van der Wijk T, Overvoorde J, den Hertog J. The Journal of biological chemistry. 2004; 279:4435544361. [PubMed: 15294898]

8. Barrett WC, DeGnore JP, Keng YF, Zhang ZY, Yim MB, Chock PB. The Journal of biological chemistry. 1999; 274:34543-34546. [PubMed: 10574916]

9. Bhattacharya S, Labutti JN, Seiner DR, Gates KS. Bioorganic \& medicinal chemistry letters. 2008; 18:5856-5859. [PubMed: 18595691]

10. LaButti JN, Chowdhury G, Reilly TJ, Gates KS. Journal of the American Chemical Society. 2007; 129:5320-5321. [PubMed: 17411049]

11. Lane AE, Tan JT, Hawkins CL, Heather AK, Davies MJ. The Biochemical journal. 2010; 430:161169. [PubMed: 20528774]

12. Kimura K, Takada M, Ishii T, Tsuji-Naito K, Akagawa M. Free radical biology \& medicine. 2012; 53:1239-1251. [PubMed: 22824864]

13. Zhou H, Singh H, Parsons ZD, Lewis SM, Bhattacharya S, Seiner DR, LaButti JN, Reilly TJ, Tanner JJ, Gates KS. Journal of the American Chemical Society. 2011; 133:15803-15805. [PubMed: 21913686]

14. Kuban-Jankowska A, Tuszynski JA, Winter P, Gorska M, Knap N, Wozniak M. PloS one. 2012; 7:e52495. [PubMed: 23300686]

15. Hsu MF, Meng TC. The Journal of biological chemistry. 2010; 285:7919-7928. [PubMed: 20064934]

16. Meng FG, Zhang ZY. Biochimica et biophysica acta. 2013; 1834:464-469. [PubMed: 22819876]

17. Conrad M, Sandin A, Forster H, Seiler A, Frijhoff J, Dagnell M, Bornkamm GW, Radmark O, Hooft van Huijsduijnen R, Aspenstrom P, Bohmer F, Ostman A. Proceedings of the National Academy of Sciences of the United States of America. 2010; 107:15774-15779. [PubMed: 20798033]

18. Meng TC, Fukada T, Tonks NK. Molecular cell. 2002; 9:387-399. [PubMed: 11864611]

19. Lee SR, Kwon KS, Kim SR, Rhee SG. The Journal of biological chemistry. 1998; 273:1536615372. [PubMed: 9624118]

20. Mahadev K, Zilbering A, Zhu L, Goldstein BJ. The Journal of biological chemistry. 2001; 276:21938-21942. [PubMed: 11297536]

21. Kwon J, Qu CK, Maeng JS, Falahati R, Lee C, Williams MS. The EMBO journal. 2005; 24:23312341. [PubMed: 15933714]

22. Singh DK, Kumar D, Siddiqui Z, Basu SK, Kumar V, Rao KV. Cell. 2005; 121:281-293. [PubMed: 15851034]

23. Giannoni E, Chiarugi P, Cozzi G, Magnelli L, Taddei ML, Fiaschi T, Buricchi F, Raugei G, Ramponi G. The Journal of biological chemistry. 2003; 278:36763-36776. [PubMed: 12815062]

24. Paulsen CE, Carroll KS. Chemical reviews. 2013; 113:4633-4679. [PubMed: 23514336] 
25. Meng TC, Buckley DA, Galic S, Tiganis T, Tonks NK. The Journal of biological chemistry. 2004; 279:37716-37725. [PubMed: 15192089]

26. Capasso M, Bhamrah MK, Henley T, Boyd RS, Langlais C, Cain K, Dinsdale D, Pulford K, Khan M, Musset B, Cherny VV, Morgan D, Gascoyne RD, Vigorito E, DeCoursey TE, MacLennan IC, Dyer MJ. Nature immunology. 2010; 11:265-272. [PubMed: 20139987]

27. Karisch R, Neel BG. The FEBS journal. 2013; 280:459-475. [PubMed: 22577968]

28. Wu RF, Terada LS. Science's STKE: signal transduction knowledge environment. 2006; 2006:pl2.

29. Boivin B, Zhang S, Arbiser JL, Zhang ZY, Tonks NK. Proceedings of the National Academy of Sciences of the United States of America. 2008; 105:9959-9964. [PubMed: 18632564]

30. Meng TC, Hsu SF, Tonks NK. Methods. 2005; 35:28-36. [PubMed: 15588983]

31. Persson C, Sjoblom T, Groen A, Kappert K, Engstrom U, Hellman U, Heldin CH, den Hertog J, Ostman A. Proceedings of the National Academy of Sciences of the United States of America. 2004; 101:1886-1891. [PubMed: 14762163]

32. Persson C, Kappert K, Engstrom U, Ostman A, Sjoblom T. Methods. 2005; 35:37-43. [PubMed: 15588984]

33. Karisch R, Fernandez M, Taylor P, Virtanen C, St-Germain JR, Jin LL, Harris IS, Mori J, Mak TW, Senis YA, Ostman A, Moran MF, Neel BG. Cell. 2011; 146:826-840. [PubMed: 21884940]

34. Benitez LV, Allison WS. The Journal of biological chemistry. 1974; 249:6234-6243. [PubMed: 4371119]

35. Brewer TF, Garcia FJ, Onak CS, Carroll KS, Chang CJ. Annual review of biochemistry. 2015; 84:765-790.

36. Leonard SE, Garcia FJ, Goodsell DS, Carroll KS. Angewandte Chemie. 2011; 50:4423-4427. [PubMed: 21504031]

37. Garcia FJ, Carroll KS. European journal of medicinal chemistry. 2014; 88:28-33. [PubMed: 24974258]

38. Seo YH, Carroll KS. Proceedings of the National Academy of Sciences of the United States of America. 2009; 106:16163-16168. [PubMed: 19805274]

39. Haque A, Andersen JN, Salmeen A, Barford D, Tonks NK. Cell. 2011; 147:185-198. [PubMed: 21962515]

40. Gupta V, Carroll KS. Chemical Science. 2016; doi: 10.1039/C5SC02569A

41. Ruddraraju KV, Parsons ZD, Llufrio EM, Frost NL, Gates KS. J Org Chem. 2015; doi: 10.1021/ acs.joc.5b01949

42. Paulsen CE, Truong TH, Garcia FJ, Homann A, Gupta V, Leonard SE, Carroll KS. Nature chemical biology. 2012; 8:57-64. [PubMed: 22158416]

43. Denu JM, Tanner KG. Biochemistry. 1998; 37:5633-5642. [PubMed: 9548949]

44. Truong TH, Garcia FJ, Seo YH, Carroll KS. Bioorganic \& medicinal chemistry letters. 2011; 21:5015-5020. [PubMed: 21601453]

45. Yang J, Gupta V, Carroll KS, Liebler DC. Nature communications. 2014; 5:4776.

46. Seo YH, Carroll KS. Angewandte Chemie. 2011; 50:1342-1345. [PubMed: 21290508] 
A Radiolabelled

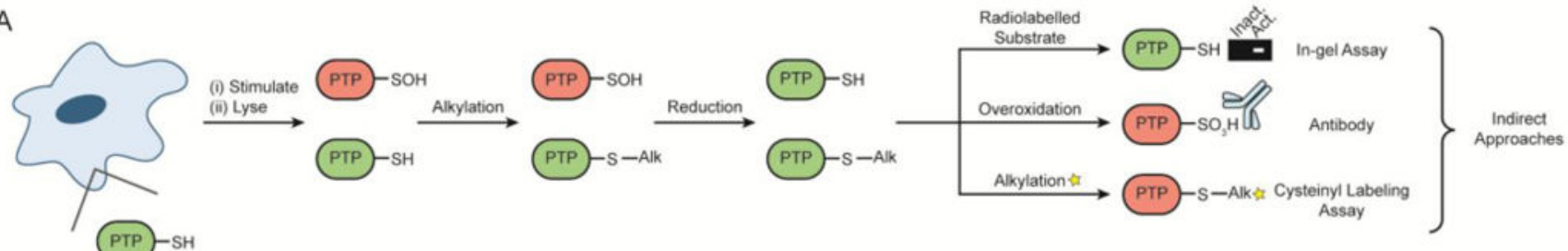

B

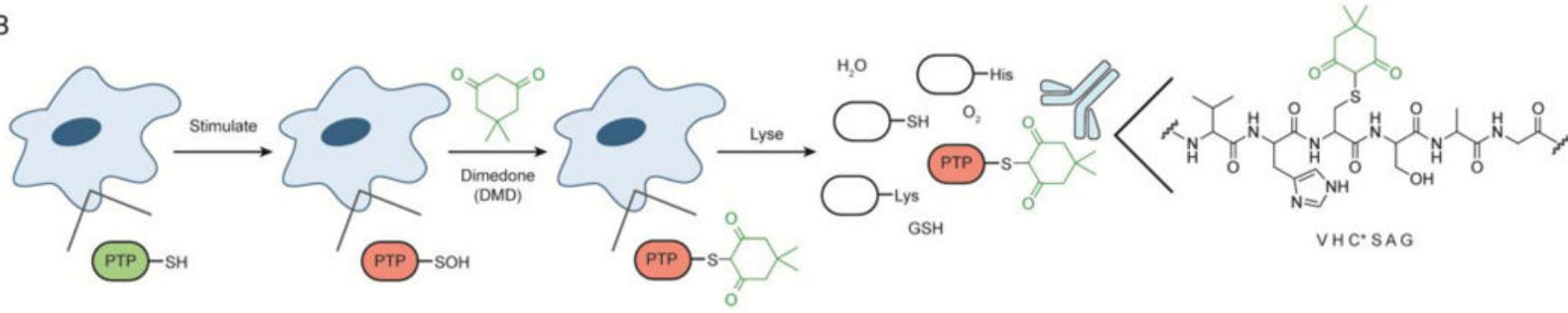

Figure 1.

Approaches available to monitor PTP oxidation. (A) Indirect approaches include in-gel activity assays that monitor protein activity via radiolabeled substrate, antibodies that recognize a unique epitope consisting of the conserved signature motif within the active site of PTPs harboring the terminally oxidized catalytic cysteine residue, and tagged thiolalkylating reagents. (B) A novel immunochemical approach, which utilizes nucleophiles such as DMD that chemoselectively react with protein sulfenic acids, to recognize the conserved catalytic motif of all PTPs bearing the DMD-thioether epitope. 
Class I PTPs

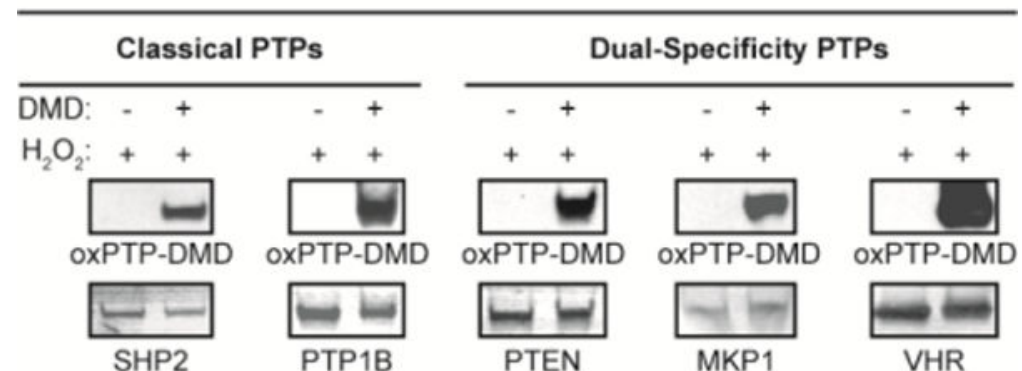

Class II PTPs

Class III PTPs

DMD: - +

$\mathrm{H}_{2} \mathrm{O}_{2}:++$

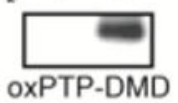

DMD: - +

$\mathrm{H}_{2} \mathrm{O}_{2}$ :
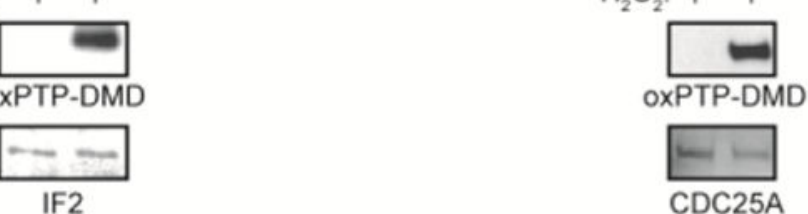

Figure 2.

Profiling oxidized PTPs. Recombinant PTPs were treated with 10 equivalences of $\mathrm{H}_{2} \mathrm{O}_{2}$ in the presence of $10 \mathrm{mM}$ DMD then analyzed by Western blot. The antibody is able to recognize the conserved PTP catalytic sequence harboring the DMD modification. 
A

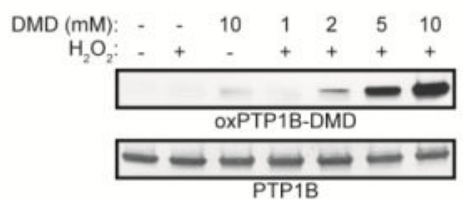

B

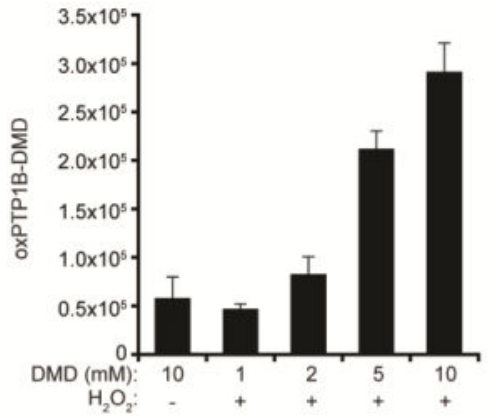

C

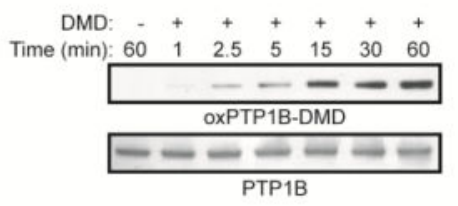

D

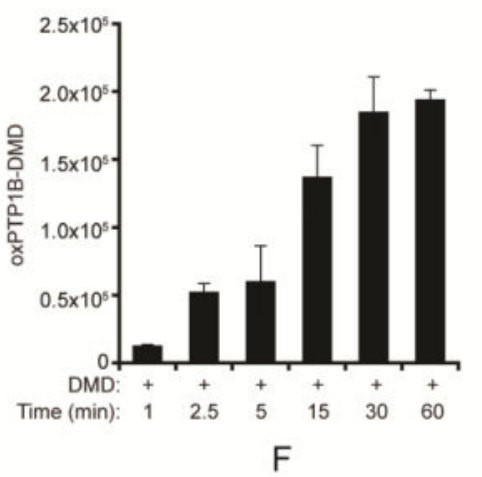

E

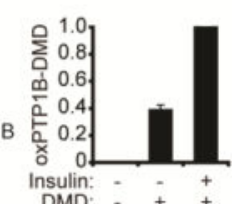

Figure 3.

The PTP-S-DMD antibody recognizes oxidized phosphatases. Recombinant PTP1B $(10 \mu \mathrm{M})$ was treated with 10 equivalences $(100 \mu \mathrm{M}) \mathrm{H}_{2} \mathrm{O}_{2}$ in the presence of increasing concentrations of DMD and Western blot analysis revealed (A) does-dependent and (C) time-dependent enhancement of DMD-modified oxPTP1B in the presence of $5 \mathrm{mM}$ DMD. Densitometric analysis of (B) dose-dependent and (D) time-dependent detection of recombinant oxPTP1B. (E) Increased levels of oxPTP1B were detected when HA-PTP1B transfected CHO/hIRc cells were stimulated with $100 \mathrm{nM}$ insulin in the presence of $10 \mathrm{mM}$ DMD. HC = heavy chain of IgG. (F) Densitometric analysis revealed a two-fold enhancement in levels of oxPTP1B after insulin stimulation. 
A

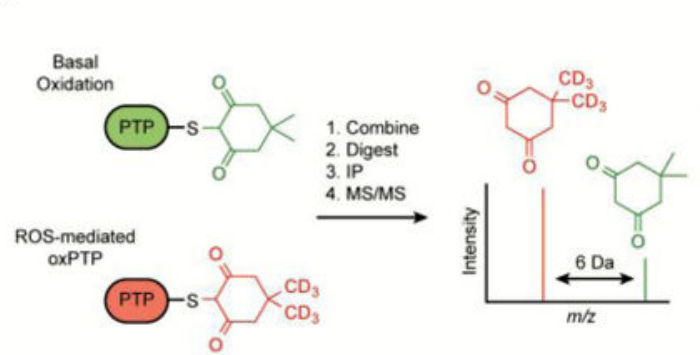

B

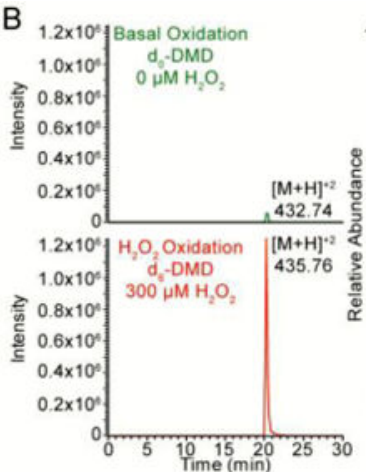

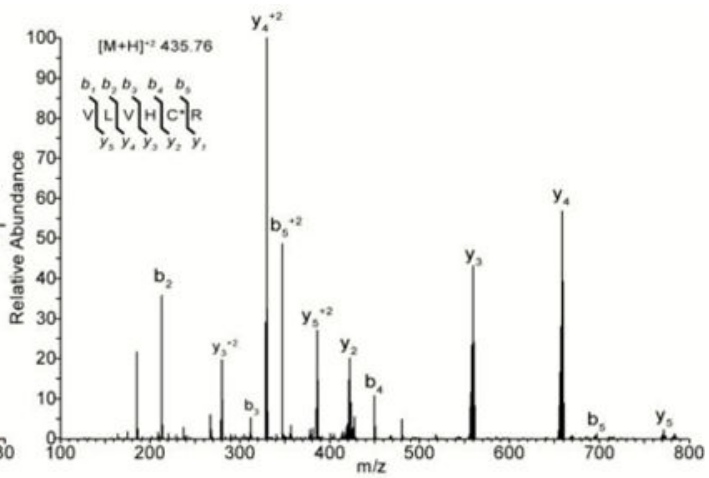

Figure 4.

Immunoaffinity enrichment of DMD-modified oxPTP peptides for quantitative proteomic analysis. (A) The use of isotopic analogs of DMD, followed by immunoaffinnity enrichment of the DMD-modified PTP peptides, and subsequent mass spectrometry analysis provides a convenient method by which to identify and quantify oxPTPs. (B) Recombinant VHR was treated with $10 \mathrm{mM} \mathrm{d}_{0}$-DMD in the absence of $\mathrm{H}_{2} \mathrm{O}_{2}$ and $10 \mathrm{mM} \mathrm{d}_{6}$-DMD in the presence of $300 \mu \mathrm{M} \mathrm{H}_{2} \mathrm{O}_{2}$. After separate incubations, the samples were mixed, digested with trypsin, enriched, and subjected to MS/MS analysis. MS/MS analysis of $435.76[\mathrm{M}+\mathrm{H}]^{+2}$ corresponds to $\mathrm{d}_{6}$-DMD modified oxVHR and displayed excellent coverage. Based on these results, we observed $\sim 5.3 \%$ basal oxVHR using this technique. 
A

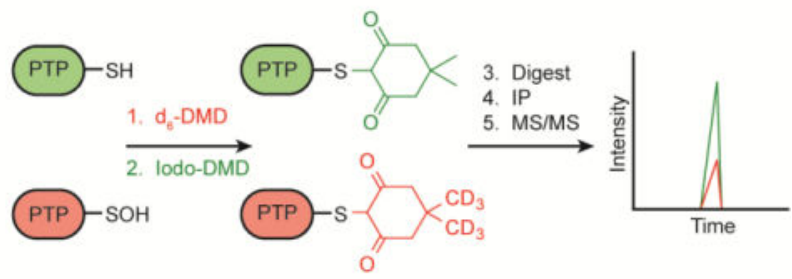

B
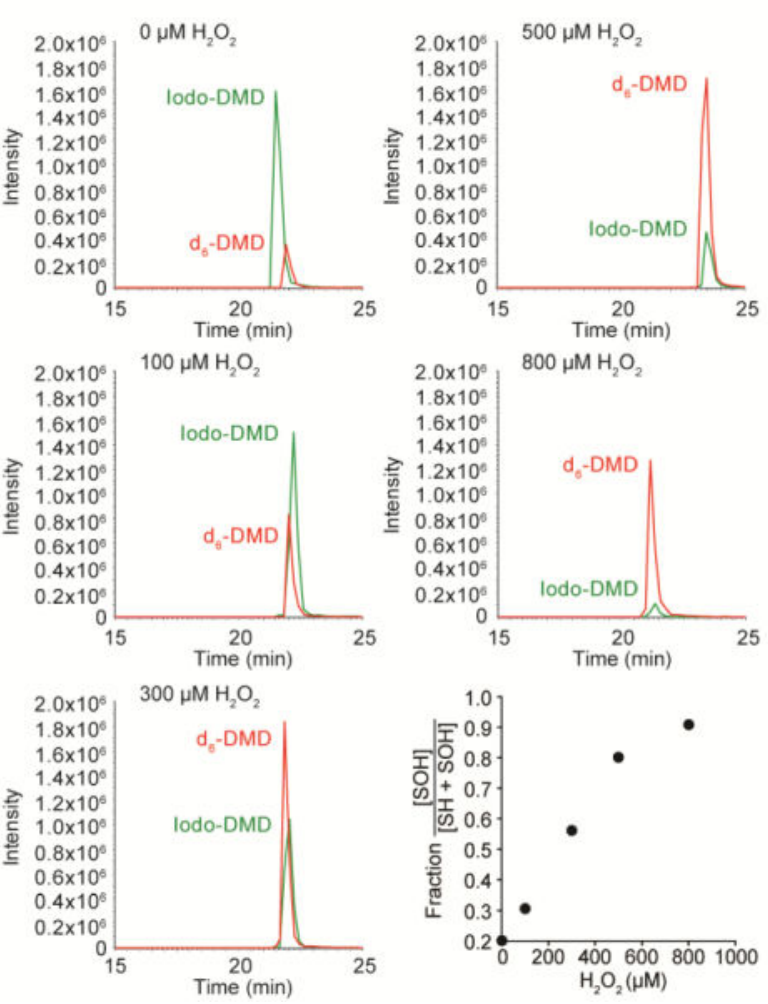

Figure 5.

Quantification of oxPTPs using the Isotope-Coded Dimedone and Iododimedone (ICDID) strategy. (A) The ICDID strategy uses a set of probes that generate chemically identical proteins but differ in the specific mass of their labels depending on the original redox state of the cysteine residue. (B) The ICDID approach provides a means for quantitative profiling and comparison of thiol redox states in physiological and pathological conditions associated with oxidative stress. We observed iododimedone modified VHR at $\mathrm{m} / \mathrm{z} 432.74[\mathrm{M}+\mathrm{H}]^{+2}$ and $\mathrm{d}_{6}$-DMD modified oxVHR at $\mathrm{m} / \mathrm{z} 435.76[\mathrm{M}+\mathrm{H}]^{+2}$ and the peaks were confirmed by MS/MS. The elution time of the light- and heavy-tagged peptide was approximately $\sim 22$ minutes. However, in some cases, small shifts in retention time were observed owing to slight variations in mobile phase, sample constituents, and/or column temperature. 